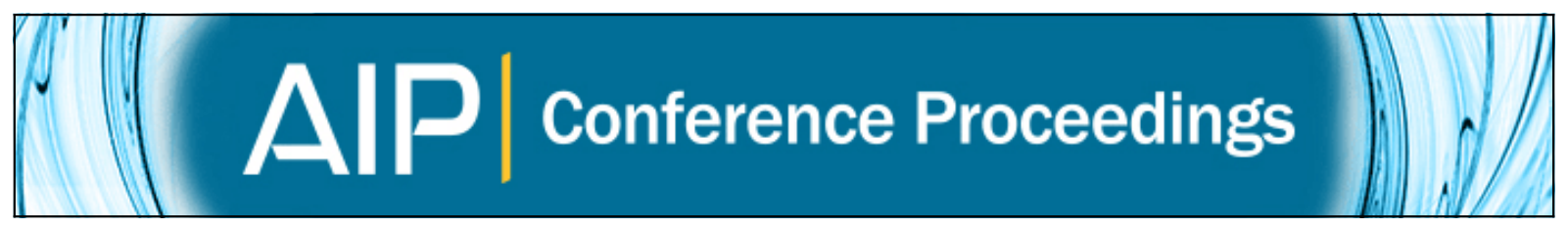

\title{
Dispersive Effects of Hall Electric Field in Turbulence
}

W. H. Matthaeus, S. Servidio, and P. Dmitruk

Citation: AIP Conference Proceedings 1216, 184 (2010); doi: 10.1063/1.3395832

View online: http://dx.doi.org/10.1063/1.3395832

View Table of Contents: http://scitation.aip.org/content/aip/proceeding/aipcp/1216?ver=pdfcov

Published by the AIP Publishing

\section{Articles you may be interested in}

Structure transitions induced by the Hall term in homogeneous and isotropic magnetohydrodynamic turbulence Phys. Plasmas 21, 072313 (2014); 10.1063/1.4890857

The Effect of Intermittent Turbulence on Cosmic-ray Transport

AIP Conf. Proc. 1366, 30 (2011); 10.1063/1.3625586

Whistler Waves Driven by Anisotropic Strahl Velocity Distributions: Cluster Observations

AIP Conf. Proc. 1216, 265 (2010); 10.1063/1.3395852

Global Solar Wind Structure: Effects of Turbulence Transport and Heating

AIP Conf. Proc. 1216, 206 (2010); 10.1063/1.3395837

Magnetic field line transport in the solar wind anisotropic turbulence

AIP Conf. Proc. 471, 547 (1999); 10.1063/1.58692 


\title{
Dispersive Effects of Hall Electric Field in Turbulence
}

\author{
W. H. Matthaeus*, S. Servidio ${ }^{\dagger}$ and P. Dmitruk ${ }^{* *}$ \\ ${ }^{*}$ Bartol Research Institute and Department of Physics \& Astronomy, University of Delaware, Newark DE 19716 \\ ${ }^{\dagger}$ Dipartimento di Fisica, Universita’ della Calabria, Ponte P. Bucci Cubo 31C, Rende, CS 87036, Italy \\ ${ }^{* *}$ Departimento de Física (FCEN-UBA), Buenos Aires, Argentina
}

\begin{abstract}
.
A familiar feature of turbulence in a low collisionality turbulence is an increase in the electric field spectrum, relative to the magnetic field spectrum, at wavenumbers near the reciprocal of the ion inertial scale. This effect is commonly observed in the solar wind. Here we examine this feature numerically, using a variety of simulations, including compressible Hall MHD, incompressible Hall MHD, and one-, two-, and three-dimensional cases. A feature of this type is even found in a statistical Hall MHD model with no dissipation. This leads to the conclusion that the only requirement for obtaining this dispersive effect is the Hall term in the generalized Ohm's law. Therefore this observation does not distinguish between whistler and kinetic Alfvén waves, between waves and turbulence, nor even between fluid and kinetic plasma models.
\end{abstract}

\section{INTRODUCTION}

In this article we review and extend recent discussion [17] of the observed enhanced of electric field spectra relative to magnetic field spectra in the solar wind, and the associated implications for plasma dissipation process in the collisionless regime.

Although diagnostics of the electric field at higher frequencies have become routinely available relatively recently [1], the roots of this subject have been studied for quite some time. Its basis rests on the observations that the fluctuation properties of the interplanetary plasma, which appear to be well described by MHD at larger scales [8] change fairly abruptly near a few Hertz in the spacecraft frame at $1 \mathrm{AU}$. First noted was the steepening of magnetic spectra near this scale [9], suggesting kinetic effects, as the scale of the steeping was found to be not far from the proton gyrofrequency. Later this was confirmed and additional properties such as magnetic helicity and anisotropy $[10,11]$ were found to change at scales smaller than the steepening scale. A more detailed analysis of the steeping scale [12] found evidence that it is more associated with the proton inertial scale $c / \omega_{p i}=V_{A} / \Omega \equiv \rho_{p}$ than it is with the gyrofrequency or the parallel resonant wave number. Furthermore the best fit to the observed break-points were obtained under the assumption that the related structures are at an angle that is highly oblique to the local mean magnetic field. This is suggestive of current sheets, reconnection activity and a very inhomogeneous heating mechanism.

Another line of reasoning to explain the steepening and associated kinetic effects is to assume that the solar wind plasma, even though highly nonlinear and turbulent at the MHD fluid scales, is described by the linear Vlasov equation when it comes to dissipation and kinetic effects.
Analysis based upon linear Vlasov theory has proceeded along these lines $[5,7,13,14]$.

In this paper we present further discussion of the possibilities for explaining the observed spectral signatures in the solar wind at the onset of kinetic effects near the ion inertial scale, emphasizing the crucial role of the Hall effect electric field.

\section{DESCRIPTIONS IN TERMS OF LINEAR VLASOV THEORY}

Earlier solar wind studies (e.g., [12, 13]) evaluated both nonlinear and linear, as well as homogeneous and inhomogeneous, models to explain the spectral steepening that terminates the MHD inertial range near the ion inertial scale. However the remark made by Bale et al [1] that the steepening might be due to Kinetic Alfvén Waves (KAWs) (note the label "KAW?" in the caption of their Fig. 3.) seems to have triggered a flurry of activity that assumes the explanation must lie in linear theory and linear wave modes.

This is perhaps best exemplified by a recent paper [7] that presents extremely important extensions of magnetic spectra from Cluster to scales smaller than the electron inertial scale $\rho_{e}$. For the first time one can see the approximate powerlaw over the full kinetic steepened scale $k \rho_{p}>1>k \rho_{e}$. For $k \rho_{e}>1$ there is further steepening suggestive of dissipation at electron scales. The paper appears to rule out by assumption there is a fraction of the energy lost at $k \rho_{p} \sim 1$ which could be due to gyroresonance since the plasma beta is $\approx$ unity. Indeed there is wealth of evidence, not only in anisotropic temperatures (e.g., [15]) but also in magnetic helicity signatures 
that suggest involvement of cyclotron-related processes in energy decay, at perhaps an approximately $50 \%$ level $[10,16]$. However the particular aspect of the work by Sahraoui et al [7] that we want to emphasize here is the conclusion, based on linearized Vlasov theory, that the wave mode that is active between the two inertial scales is the Kinetic Alfven Wave. The evidentiary basis for this is mainly that the observed ratio of electric field spectrum to magnetic field spectrum $E / B \sim k$ in the range $k \rho_{p}>1>k \rho_{e}$. In the next section we show that this does not, in general, imply only KAW. However if one makes the additional assumption (apparently made in [7] but not stated) that the system must be described by linear Vlasov theory, then the KAW conclusion may stand on firmer ground. This argument appears to be rather incomplete since it tacitly rules out all nonlinear process, as well as all inhomogeneous processes, including those involving current sheets, reconnection, and strong kinetic turbulence that might heat the plasma, but which does not satisfy a linear dispersion relation.

A similar argument, with similar weaknesses, was presented in a numerical demonstration of the gyrokinetic equations [5]. Here, the authors have assumed at the onset, by adopting gyrokinetics, that the system of interest (the solar wind) must fit into that restricted context. Thus, whistlers are ruled out, for example. In developing an interpretation, these authors also describe the increasing ratio $E / B$ of spectra as "compelling evidence that the observed breaks in the spectra are caused by a transition to a KAW cascade..." Again, this evidence does not support such a strong conclusion (see [3]): their assertion is not ruled out - but it is also not implied.

We cite the above papers as examples of what we think is incomplete reasoning regarding the solar wind spectral features of interest. We will now turn to evidence that shows the above conclusions, based on restrictive but tacit assumptions, do not follow from the "dispersive" increase in $E / B$ spectra cited above [1].

\section{PROSPECTS FOR A TURBULENCE EXPLANATION}

As we mentioned above, various studies have offered explanations for steepening and dissipation in solar wind spectra at and above the wavenumber $k \rho_{p}>1[6,11$, $12,16,17]$. Other processes have been suggested that are fundamentally inhomogeneous and nonlinear [18]. Further observational analysis points towards the requirement that the relevant processes (suggested to be necessarily more than one) must be nonlinear [19].

With this complex observational background in mind, one can also ask the relatively simple question: Does the increase in the ratio of electric field spectrum to magnetic
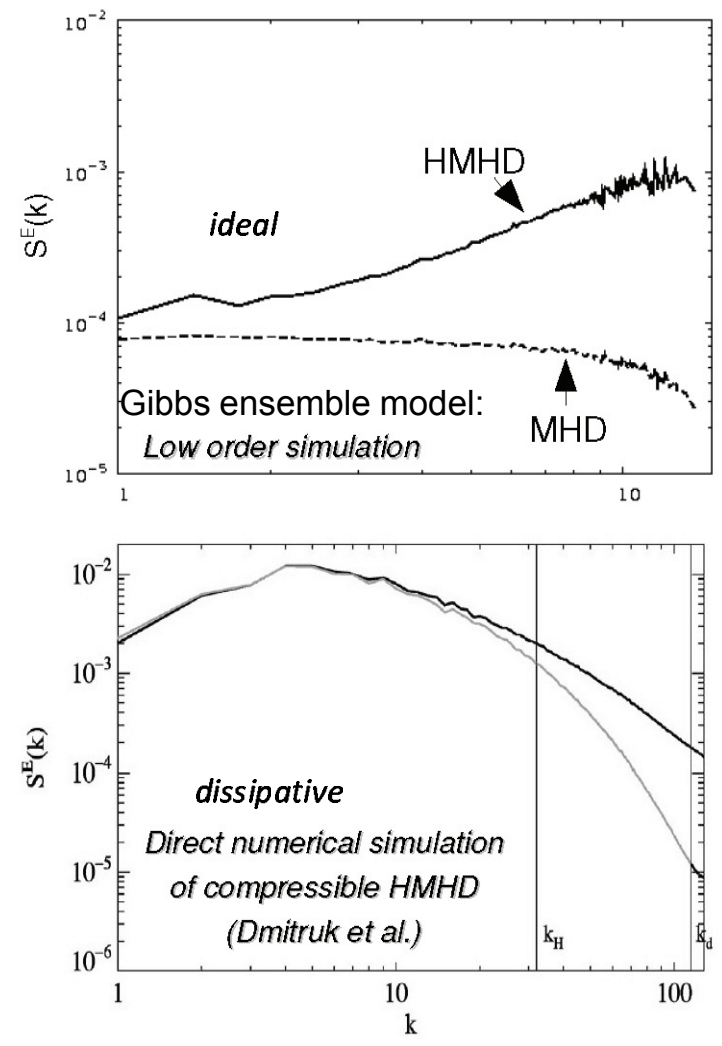

FIGURE 1. Electric field wavenumber spectra. (Top) Two ideal Gibbs ensemble simulations (no dissipation), ordinary MHD (dashed line) and Hall MHD (solid line). (Bottom)Dissipative 3D spectral method simulations, ordinary MHD (gray line) and Hall MHD (black line). In both cases the presence of the Hall effect gives rise to enhancements of electric field spectra at high wavenumbers.

field spectrum imply anything in particular about the nature of the fluctuations involved?

In an earlier commentary [3] on the above discussed gyrokinetics paper [5] we addressed this question by providing examples of enhanced spectral ration $E / B$ in several types of simulations. In particular, this increase in spectral ratios above $k \rho_{p}=1$ is seen in

- 3D compressible Hall simulations with a mean field

- 2D compressible Hall simulations with a mean field

- 1D compressible Hall simulations with a mean field

- 3D ideal incompressible simulations without a mean field

- 3D compressible simulations without a mean field

- an analytical Gibbs ensemble spectral prediction for ideal 3D Hall MHD [20]

After consideration of these results, it becomes apparent that all that is needed to see this effect is the presence 
of the Hall effect in Ohm's law. Even if this is sometimes called a "dispersion" effect, no linear dispersion relation is implied, and no particular wave mode, either linear or nonlinear is implied. In fact it does not even require a mean magnetic field, or even dissipation of any kind, to be present. For the structure of Ohm's Law, see the Appendix.

To clarify this result even more, we show in Figures 1-3 descriptions of electric and magnetic spectra from several types of simulations, including (Fig. 1) ideal spectral method simulations (with results described by an equilibrium Gibbs ensemble), (Fig. 2) 3D and (Fig. 3) $2.5 \mathrm{D}$ compressible simulations. Fig. 1 compares the electric field spectrum seen in a Gibbs ensemble model (with no mean field) for MHD (no Hall effect) with Hall MHD (with Hall effect). The Hall term is clearly responsible for enhanced electric field at high $k$. For the 3D case shown in Fig. 2, the code is a pseudospectral compressible Hall code, with a resolution of $256^{3}$ spatial points, and a value of Hall wavenumber $k_{h}=32$ in units of the largest wavelength. For the $2.5 \mathrm{D}$ case in Fig. 3, the code uses fourth order finite difference, with mean field $B_{0}=1$ and $k_{h}=80$. The allegedly kinetic dispersive ratio of $E / B \sim k^{1}$ is seen in both cases. However many types of "modes" might be present in the 3D case, which is only moderately anisotropic, while usual oblique KAW are absent in the $2.5 \mathrm{D}$ case since $k_{\|}=0$

\section{DISCUSSION AND CONCLUSION}

We have reviewed observational and theoretical evidence that is relevant to the nature of the spectral steepening seen in the solar wind near the ion inertial scale. We see little reason at this point to rule out contribution to steepening and heating due to a number of possible processes, at least some of which are nonlinear and/or inhomogeneous. Therefore we contest recent claims made in various studies that argue that observations and theory necessarily point exclusively to Kinetic Alfvén Waves (a linear, homogeneous Vlasov construct) as the "mode" that makes up solar wind fluctuations at $k \rho_{p}>1$. In this regard, the alleged "smoking gun", enhanced $E / B \sim k^{1}$ is not at all difficult to obtain, in many possible models. KAWs are not ruled out, but they are not implied.

Furthermore, we also see little real evidence that supports the restriction in our dialogue to the "wave mode" vocabulary of linear Vlasov theory. Instead we suspect that there is a substantial role for current sheets, reconnection, vortices and zero-frequency turbulence in the explanation of spectral features and heating in the solar wind plasma and related solar and astrophysical plasmas.

This research supported in part by the NSF SHINE (ATM0752135) and solar-terrestrial (ATM0539995) programs, and by the NASA Heliophysics Theory Program
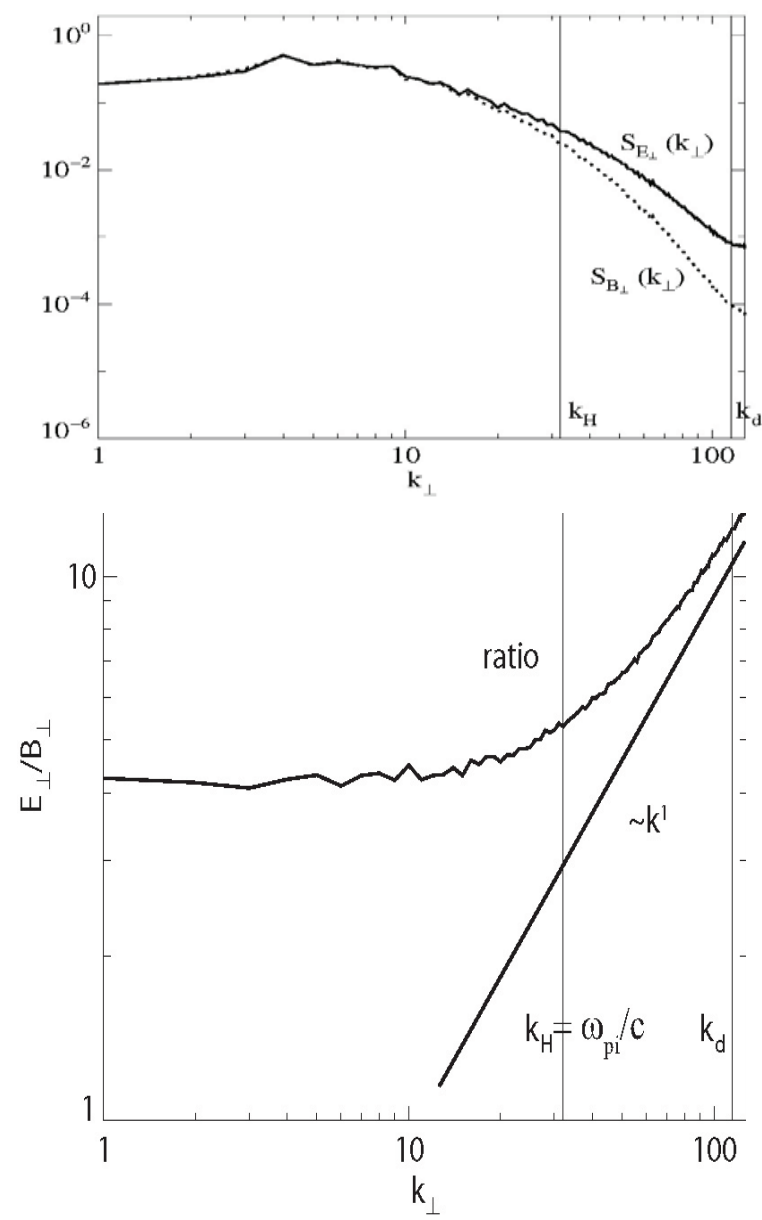

FIGURE 2. (Top) Wavenumber ( $k$ ) spectra of the perpendicular components of the electric and magnetic fields, for a 3D Hall MHD simulation. The Hall-scale wavenumber $k_{h}$ (reciprocal ion inertial scale) is shown as a vertical line. For $k<k_{h}$, the two spectra track each other well. For $k>k_{h}$, the electric field spectrum rises above the magnetic spectrum. (Bottom) The ratio of electric over magnetic spectrum as function of $k$. For $k>k_{h}$, the ratio scales as $\sim k^{1}$ as suggested by the reference line.

(NNX08AI47G).

\section{APPENDIX: GENERALIZED OHM'S LAW}

A well known feature in low collisionality plasmas is a change in the character of the electric field near the ion inertial scale. This is most easily understood in terms of a generalized Ohm's law that can be written

$$
\mathbf{E}+\mathbf{u} \times \mathbf{B}=\eta \mathbf{J}+\frac{1}{n e c} \mathbf{J} \times \mathbf{B}-\frac{1}{n e} \nabla P_{e}+\frac{m_{e}}{n e^{2}} \frac{\partial \mathbf{J}}{\partial t}
$$




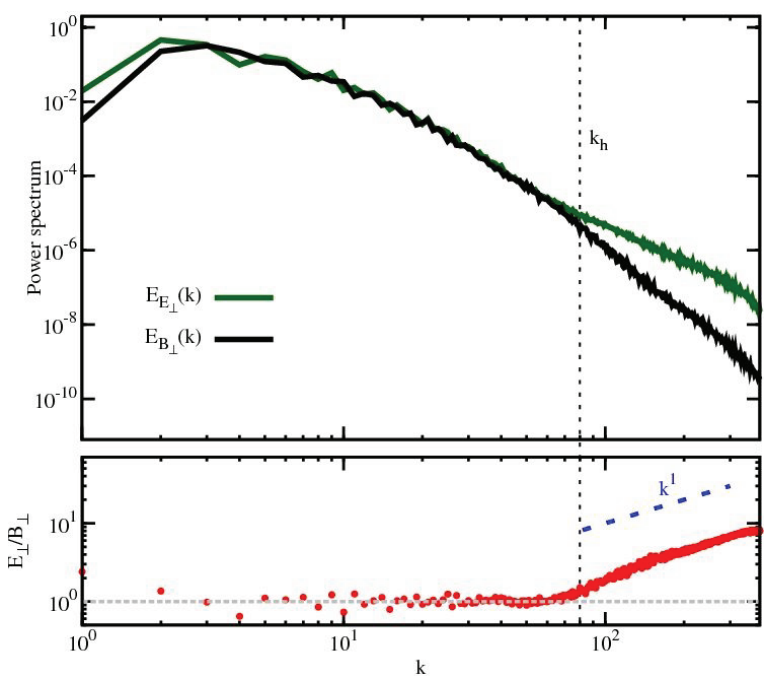

FIGURE 3. (Top) Similar format to Fig. 2, but for a 2.5D compressible Hall MHD simulation. The spectra again track each other for $k<k_{h}$ but the electric spectrum become greater than the magnetic spectrum at $k>k_{h}$. (Bottom) The ratio of electric to magnetic spectra. Again, for $k>k_{h}$ the ration scales as $\sim k^{1}$.

The first term on the r.h.s. is the classical collisional resistive dissipation with resistivity $\eta$. With proper averaging, the effects of small scale turbulence can be included in this term as a "turbulent resistivity." Next is the Hall term $\mathbf{J} \times \mathbf{B}$, involving current density $\mathbf{J}$ and magnetic field $\mathbf{B}$. This term is associated with differential flow of ions and electrons, and becomes appreciable at the ion inertial scale $\rho_{i i}=c / \omega_{p i}$. The electron pressure $P_{e}$ term is of the order of $\beta_{e} \rho_{i i}$ (electron plasma beta $\beta_{e}$, the ratio of electron pressure to magnetic pressure.) The final term in Eq. (1) is due to electron dynamics and is appreciable at the electron inertial scale $c / \omega_{p e}$. If the right hand side vanishes, the "ideal" MHD Ohm's law emerges, $\mathbf{E}+\mathbf{u} \times \mathbf{B}=0$, in which the magnetic flux is "frozen-in" the bulk plasma, moving at the center of mass fluid velocity $\mathbf{u}$. The electron pressure term is, more precisely, the divergence of a pressure tensor (i.e. $\nabla \cdot \mathbf{P}_{\mathbf{e}}$ ).

\section{REFERENCES}

1. S. D. Bale, et al., Phys. Rev. Lett. 94, 215002 (2005).

2. P. Dmitruk \& W. H. Matthaeus, Phys. Plasmas 13, 2307 (2006).

3. W. Matthaeus et al, PRL, 101, 149501 (2008).

4. S. Saito, et al., Phys. of Plasmas, 15, 102305 (2008).

5. G. Howes et al, PRL, 100, 065004 (2008).

6. J. P. Eastwood et al, PRL, 102, 035001 (2009).

7. F. Sahraoui et al, Phys. Rev. Lett., 102, 231102 (2009).

8. Barnes, A., in Solar System Plasma Physics, Vol. 1, ed. E. N. Parker, C. F. Kennel, \& L. J. Lanzerotti (1979).
9. Denskat, K. U., Beinroth, H. J., \& Neubauer, F. M., J. Geophys., 54, 60 (1983).

10. Goldstein, M. L., Roberts, D. A., \& Fitch, C. A., J. Geophys. Res., 99, 11519 (1994).

11. Leamon, R. J., Smith, C. W., Ness, N. F., Matthaeus, W. H., \& Wong, H. K., J. Geophys. Res., 103, 4775 (1998).

12. Leamon, R. J., et al., Astrophys. J., 537, 1054 (2000).

13. Leamon, R. J., Smith, C. W., Ness, N. F., \& Wong, H. K. J. Geophys. Res., 104, 22331 (1999).

14. Gary, S. P., \& Borovsky, J. E. J. Geophys. Res. (Space Physics), 113, 12104 (2008).

15. Marsch, E., R. Schwenn, H. Rosenbauer, K-H., Muehlhaeuser, K-H. Pilipp and F. M. Neubauer, J. Geophys. Res. 87, 52 (1982).

16. Leamon, R. J., W. H. Matthaeus, C. W. Smith and H. K. Wong, Astrophys. J., 507, L181 (1998).

17. Sundkvist, D., A. Retino, A. Vaivads, and S. Bale, Phys. Rev. Lett., 99, 025004 (2007).

18. Markovskii, S., B. J. Vasquez, C. W. Smith and J. V. Hollweg, Astrophys. J., 639, 1177 (2006).

19. Markovskii, S. ,B. J. Vasquez, and C. W. Smith, Astrophys. J., 675, 1576 (2008).

20. Servidio, S., W. H. Matthaeus, and V. Carbone, Phys. Plasmas, 15, 042314 (2008). 